Z Rheumatol 2020 $\cdot 79: 497$

https://doi.org/10.1007/s00393-020-00813-3

Online publiziert: 19 . Mai 2020

(c) Springer Medizin Verlag GmbH, ein Teil von Springer Nature 2020

Prof. Dr. med. Norbert Gschwend, geb. am 29.08.1925, ist am 22.03.2020 von uns gegangen. Er war zu einer Zeit, in der die „Rheumatoide Arthritis“ (RA) noch „primär chronische Polyarthritis“ (PCP) hieß, einer der bedeutendsten Pioniere der orthopädischen Rheumatologie.

Als Gründungsmitglied und Präsident der Assoziation für Orthopädische Rheumatologie (ARO), heute Deutsche Gesellschaft für Orthopädische Rheumatologie (DGORh), prägte er die Entwicklung der jungen Fachgesellschaft und der operativen Therapie entzündlich rheumatischer Erkrankungen entscheidend. Die von ihm zusammen mit H.J.G. Scheier und A. Bähler an der Schulthess Klinik in Zürich entwickelten GSB-Knie- und Ellenbogengelenkprothesen fanden weltweite Verbreitung zur Behandlung instabiler destruierter Gelenke und ermöglichten so zahllosen Patientinnen und Patienten eine Verbesserung ihrer Lebensqualität und den Erhalt oder die Wiederherstellung ihrer Selbstständigkeit.

Bereits in den 70er Jahren, Jahrzehnte vor der Entstehung der Endoprothesenregister, untersuchte Prof. Gschwend mit seinem Team Knie- und Hüftprothesen systematisch nach. Er war damit seiner Zeit auch in dieser Hinsicht weit voraus.

Seine zahlreiche Publikationen zur operativen Therapie rheumatischer Krankheitsbilder in nationalen und internationalen Fachzeitschriften sowie seine Bücher waren richtungsweisend für orthopädische Rheumatologen in der ganzen Welt. Das von Norbert Gschwend seit 1968 in mehreren überarbeiteten Auflagen verfasste Lehrbuch zur operativen Behandlung der chronischen Polyarthritis war das erste deutschsprachige Standardwerk zu die-

Vorstände der Deutschen Gesellschaften für Rheumatologie und Orthopädische Rheumatologie, Berlin

\title{
Rheumatologen trauern um Prof. Norbert Gschwend
}

sem Thema und somit Grundlage für die operative Ausbildung von Generationen orthopädischer Rheumatologinnen und Rheumatologen im deutschsprachigen Raum.

Unter der Leitung von Norbert Gschwend wurde die bis dahin weithin unbekannte Schulthessklinik in Zürich zu einer der weltweit bekanntesten Kliniken für Orthopädie, Endoprothetik und orthopädische Rheumatologie, deren Ruf auch heute noch besteht und eng mit seinem Namen verbunden ist. Um eine ganzheitliche Versorgung der Rheumapatienten $\mathrm{zu}$ ermöglichen, etablierte er sehr früh Abteilungen für Rheumatologie, Neurologie sowie Ergo- und Physiotherapie an seiner Klinik. Norbert Gschwend habilitierte sich 1968 mit dem Thema „Die operative Behandlung der progredient chronischen Polyarthritis“, erhielt 1969 die Venia legendi und war von 1975 bis 2000 Titularprofessor für Orthopädie an der Universität Zürich. Die zahlreichen Ehrungen, die ihm über Jahrzehnte seines unermüdlichen Schaffen zuteil wurden, zeugen von seiner überragenden Persönlichkeit und Innovationskraft.

Die Deutsche Gesellschaft für Orthopädische Rheumatologie und die Deutschen Gesellschaft für Rheumatologie trauern gemeinsam um ihr Ehrenmitglied Norbert Gschwend und werden ihn in ehrendem Andenken behalten.

Die Vorstände der Deutschen Gesellschaften für Rheumatologie und Orthopädische Rheumatologie

\section{Korrespondenzadresse}

Prof. Dr. R. Gaulke

Deutsche Gesellschaft für Orthopädische Rheumatologie e.V. (DGORh)

Straße des 17. Juni 106-108, 10623 Berlin, Deutschland

gaulke.ralph@mh-hannover.de 